\title{
An Offer You Can't Refuse: Early Contracting with Endogenous Threat*
}

\author{
Bruno Jullien ${ }^{\dagger}$ Jerome Pouyet ${ }^{\ddagger}$ Wilfried Sand-Zantman ${ }^{\S}$
}

December 2016

\begin{abstract}
A seller has private information on the future gains from trade with a buyer, but the buyer has the option to invest to produce the good internally. Both the buyer and the seller can efficiently trade ex post under complete information. Despite the lack of information, the buyer sometimes gains by making an early contract offer to the seller. The early contract divides the different types of seller according to their information, which renders the threat of producing the good in-house credible and enables the buyer to extract a larger share of the gains from trade. Several extensions are investigated.
\end{abstract}

${ }^{*}$ This article supersedes the working paper "Divide and Learn: Early Contracting with Endogenous Threat." We have benefited from the detailed comments of J.E. de Bettignies, G. Celik, O. Compte, B. MacLeod, D. Martimort, M. Peitz, J. Swinkels, T. Valletti and seminar participants at Paris School of Economics (2011), 2011 CSIO/IDEI conference, Télécom ParisTech (2011), the University of Besançon (2011), the ENTER Jamboree (UAB, 2012), University College London (2012), Thema (2013), Toulouse Business School (2013), Imperial College London (2013), and at the EARIE 2012 conference in Roma. We are indebted to the editor, Benjamin Hermalin, and two referees for useful remarks on a previous draft. We also gratefully acknowledge Orange for its financial support. This work was supported by a grant overseen by the French National Research Agency (ANR-12-BSH1-0009).

†Toulouse School of Economics (CNRS); bruno.jullien@tse-fr.eu.

${ }^{\ddagger}$ Paris School of Economics (CNRS) and ENS; jerome.pouyet@ens.fr.

${ }^{\S}$ Toulouse School of Economics (Université de Toulouse) and ESSEC Business School; wsandz@tse-fr.eu. 


\section{Introduction}

Although asymmetric information has been recognized as a major impediment to efficient trade, much less is known about its impact on the bargaining positions of the trading parties. ${ }^{1}$ We show in this article that asymmetric information allows the uninformed party to use threats that would not otherwise be credible, thereby providing the uninformed party with a larger share of the trade surplus.

As an illustration, consider the following situation: a CEO must decide between using an external specialist for performing repairs or maintenance on her infrastructure or performing these tasks in-house. The likelihood of repairs depends on various elements that only this specialist may know well, in particular when the CEO has been newly appointed and therefore has poor information on the likelihood of requiring the specialist's skills or when the service is related to a new project. ${ }^{2}$

From an organizational perspective, the CEO has three options. The first is to wait and employ the specialist when needed, an ex post transaction that is assumed to take place under complete information. Another option is to produce the service internally, perhaps by dedicating resources to train a team within the firm; this is a profitable option only when the likelihood of needing the service is sufficiently high. The last option is to propose a contract to the specialist ex ante, before the realization of the firm's need, to specify a price when the specialist's service is needed. Such a contract takes place under asymmetric information between the firm and the provider, and the CEO cannot commit to the action chosen were the specialist to refuse that contract.

Our analysis reveals that the CEO may choose the last option and use the threat to rely on in-house supply as a tool to reduce the price paid to the specialist, despite the CEO's inability to commit to using this threat. Specifically, being at an informational disadvantage may increase the credibility of the option to produce the service internally, thereby providing the CEO with a stronger ex ante bargaining position vis-à-vis the specialist. When this is indeed the case, the CEO gains more

\footnotetext{
${ }^{1}$ See Laffont and Martimort (2002) for the consequences of incomplete information and the design of constrained optimal contracts.

${ }^{2}$ In the conclusion, we suggest several other applications with similar features.
} 
by proposing an ex ante contract under asymmetric information than by relying on ex post supply under complete information.

This ex ante contract exploits the heterogeneity among the different types of specialist; that is, some types know that the likelihood of repair is low, whereas other types know that it is high. Suppose that the CEO proposes a contract to the specialist. When the likelihood of the firm needing the specialist's services is low enough, the specialist accepts that contract because his expected gain from an ex post trade is low. Following a refusal, the CEO must therefore infer that likelihood is high, making the option of producing the service internally more profitable. This deters a specialist who knows that the likelihood is high from refusing the contract. There is thus an information trap whereby acceptance by certain types of specialist forces acceptance by the other types, even when the offer is less profitable for some specialists than their gain from ex post trade.

To make this point in the most transparent way, we model the relationship between a potential buyer (she - the CEO in the above illustration) and a seller (he - the specialist). The buyer will have unit demand with some probability $\theta$ that is privately known to the seller. Conditional on the demand being positive, the reservation utility of the buyer is fixed at a commonly known value. In this case, gains from trade are always realized ex post, and the only question is the division of the surplus between the buyer and the seller. Our model involves two periods, and demand is realized in the second period (ex post).

Faced with uncertainty, the buyer can wait until the demand is realized. In this case, there is a negotiation in the second period between the seller and the buyer, and the good is traded if demand is positive. During the first period, the buyer may instead decide to invest to produce the good in-house; such an investment is referred to as "bypass." Prior to that decision, the buyer can also propose a contract to the seller that specifies the future terms of trade. A feature that makes the contracting problem interesting and novel is the inability of both parties to commit to future actions in the absence of a contract.

To highlight the strength of our mechanism, we assume that, based on her prior beliefs regarding $\theta$, the buyer does not find it profitable to produce the good in-house. This means that, absent additional information, the buyer cannot credibly use her 
ability to invest as a threat to reduce the price paid ex ante. When the seller has no superior information with respect to the buyer, the only outcome is then that the buyer waits and trades ex post if the demand is positive. We show that, instead, if the seller privately knows $\theta$ in the first period and the buyer's investment cost is not too large, the buyer proposes an early contract and thereby obtains a payoff strictly above the payoff that she would obtain by waiting to trade ex post under complete information.

In the first part of the article, we fully characterize the equilibrium payoffs of the game described above when the ex ante contract offered by the buyer is restricted to a fixed price. Following a contract being refused by the seller, the buyer decides to bypass if her beliefs on the probability that her demand will be positive are sufficiently high. This interaction between the contract and the post-rejection beliefs may be the source of a multiplicity of continuation equilibria following a contract offer. The ability of the buyer to reduce the rent of the seller depends on how this multiplicity is resolved. We derive the set of equilibrium payoffs and show that the buyer's gains are higher when the seller has some information than when he has none.

In the second part of the article, we propose several extensions of our model. We first introduce the possibility that some sellers may not have any information to illustrate the role of heterogeneity in the seller's information. Early contracting emerges when a sufficiently large proportion of sellers are informed, and the buyer's gain increases with the fraction of informed sellers. Then, we endogenize the seller's decision to initially enter the market by adding an ex ante investment stage. We derive conditions for such an investment to occur. Finally, we extend the model to the case in which the buyer can propose any non-linear contract and show that this does not modify the set of equilibrium payoffs.

Our model builds on several strands of the literature.

A critical feature of our analysis is the buyer's outside option, that is, the possibility to internally produce the service rather than relying on external provision. In that respect, we build on the literature on the firm's boundaries (for a general presentation, see Williamson, 2005), but we offer a new insight: the flexibility of the firm, interpreted as the cost of producing in-house rather than utilizing an external 
supplier, can be used strategically to improve the firm's bargaining position vis-à-vis better informed suppliers.

That there is no ex ante commitment on the decision to produce in-house if the seller rejects the offer differentiates our analysis from contributions on the makeor-buy decision under asymmetric information (see, among others, Dana and Spier, 1994). This implies that the outside option becomes endogenous in our model, as it depends both on the seller's information and on the possibility that the buyer and the seller may interact subsequently even if the contract is rejected.

The general insight that the rejection of a contractual offer reveals information about the agent dates back at least to Coase's (1972) classical analysis of a durable good monopoly (see Hart and Tirole, 1988) and can also be found in the litigation literature (see Bebchuk, 1984). Our setup differs, as the contract is designed such that the information revealed by not participating is unfavorable to the agent, which makes it possible to increase the participation level. ${ }^{3}$

The idea that contract negotiation conveys information that may shape future profits and bargaining positions is also reminiscent of the work on the dynamic ratchet effect (Weitzman, 1980; Freixas, Guesnerie, and Tirole, 1985; Laffont and Tirole, 1988). In this literature, the information revealed during the course of the contractual relationship may be subsequently exploited and is detrimental from an ex ante perspective. In our model, the information is conveyed by the refusal decision, not during the relationship, and benefits the uninformed buyer. To some extent, one may regard our contribution as a case of strategic manipulation of the unraveling process highlighted by Grossman (1981) and Milgrom (1981) in the context of strategic information disclosure.

Cramton and Palfrey (1995) and Celik and Peters (2011) adopt a mechanismdesign perspective and analyze situations in which the failure to contract triggers further interactions between the parties. Cramton and Palfrey (1995) introduce the notion of ratifiability, which selects among implementable allocations using forward induction arguments along the lines of Grossman and Perry (1986). The lack of

\footnotetext{
${ }^{3}$ Recent articles on the dynamic contracting literature, such as Deb and Said (2016) and Skreta (2015), explore the consequences of limited commitment. Our mechanism differs from theirs, as all types of seller accept and our article is applied to different issues but follows a somewhat similar logic.
} 
commitment also generates multiple equilibria, as in our work, and the equilibrium we focus on corresponds to that highlighted by Cramton and Palfrey (1995).

Finally, our analysis sheds some light on the value of information in principalagent models, by exhibiting a situation in which the principal may benefit from negotiating with a better-informed agent. Under moral hazard, information is also relevant for the decision process, such that a principal may prefer dealing with an informed agent (see Sobel, 1993, and Kessler, 1998). In our analysis, there is no moral hazard, and thus, information has no direct effect on efficiency.

The article is organized as follows. Section 2 presents the basic model. Section 3 derives the equilibrium payoffs. Section 4 proposes several extensions. Section 5 discusses the robustness of our approach in a more general setting, and Section 6 concludes.

\section{Basic model}

A buyer (she) may purchase one unit of a good from a seller (he). The seller's production cost is normalized to zero. The buyer's utility derived from consumption is either 0 or 1 with probability $1-\theta$ and $\theta$, respectively. In period 1 , the seller knows $\theta$ whereas the buyer only knows its distribution, represented by a (atomless) cumulative distribution $F(\cdot)$ and a (positive) density $f(\cdot)=F^{\prime}(\cdot)$ defined over $[0,1]$.

There are two periods. In the first period (called the ex ante period), the buyer may offer the seller an early contract. If she does so, the offer is on a take-it-orleave-it basis. As only the seller knows $\theta$, contracting in the first period takes place under asymmetric information. If the seller accepts the buyer's offer, the contract is implemented. If the seller rejects the offer, the buyer has two options. In the first case, she invests an amount $k$ that allows her to produce in-house the good during the second period at no additional cost; this option is referred to as "bypass". Alternatively, the buyer waits until the second period to buy the good if needed.

At the beginning of the second period (called the ex post period), the buyer's valuation is realized. If it is positive and no early contract has been signed, the buyer and the seller enter into ex post negotiation, which we model as follows: with 
probability $\alpha$, the seller has the bargaining power and sets a price equal to 1 to extract all the buyer's surplus; with probability $1-\alpha$, the buyer has the bargaining power and sets a price equal to the seller's marginal cost, here 0 .

This specific modeling of the ex post trade is not crucial for our analysis. What really matters is that the seller receives a positive share of the surplus in the event of ex post trade. If the seller had no bargaining power ex post $(\alpha=0)$, the buyer would never lose from postponing her buying decision to period $2 .^{4}$

The equilibrium concept is Perfect Bayesian Equilibrium in pure strategies. We make the assumption that the buyer makes no early offer unless she expects a positive gain from doing so.

In the main section, we assume that the buyer's early offer takes the form of a fixed price $T$ paid in advance to obtain the good in period 2 if needed. ${ }^{5}$ Without loss of generality, we restrict attention to offer $T$ such that $0 \leq T<\alpha$, referred to as an admissible offer. Indeed, a negative offer $T$ would be rejected for certain by the seller, whereas the buyer would never make an offer $T$ that costs more than waiting for all types of seller.

As a first benchmark, assume complete information on $\theta$ in the first period. Trade with no bypass must then occur, as this maximizes social surplus. The sharing of this surplus depends on whether in-house production is a relevant threat for the buyer. This is a credible alternative when it yields an expected gain larger than the gain associated with waiting and buying the good at the ex post price, that is, when

$$
\theta-k \geq \theta(1-\alpha) \Leftrightarrow \theta \geq \frac{k}{\alpha} \equiv \theta^{B}
$$

As a consequence, we obtain the following equilibrium characterization:

- When $\theta$ is greater than $\theta^{B}$, the buyer offers a zero price in the ex ante stage, $T=0$, because in-house production is a credible threat.

\footnotetext{
${ }^{4}$ Observe that in our model, ex post trade is efficient. If such trade were not efficient, as bargaining could either fail or lead to suboptimal outcomes in a more complex environment, then the rationale for using an early contract would be even stronger. See our working paper, Jullien, Pouyet, and Sand-Zantman (2013).

${ }^{5}$ As pointed by a referee, this resembles "retainer agreements" used for legal services.
} 
- When $\theta$ is less than $\theta^{B}$, the buyer does not offer any contract in period 1 because this would require compensating the seller for the ex post profit $\theta \alpha$; trade occurs only ex post.

- In the case in which $\theta$ is equal to $\theta^{B}$, the buyer is indifferent between bypassing and not. As a result, both an accepted zero price offer and no offer are equilibria, the former occurring when the buyer bypasses after refusal and the latter occurring when the buyer never bypasses.

The same conclusion would hold, replacing $\theta$ with its expected value, $\mathbb{E}\{\theta\}$, if neither party were informed of the true value of $\theta$.

As a second benchmark, if the buyer could commit to bypass each time the seller rejects her offer, she would then be able to impose a price equal to zero regardless of the information structure. We assume instead that there is no commitment on the decision of whether to bypass, a key ingredient of our analysis.

\section{Contracting under asymmetric information}

In this section, we derive the early offer, if any, that the buyer proposes and the equilibrium payoffs of the two parties. Under asymmetric information, the seller's decision to reject an offer conveys some information to the buyer that will affect her decision of whether to bypass. We first analyze this interaction between rejection and bypass and then characterize the equilibrium offers and payoffs.

\section{Contract rejection and bypass decisions}

We first examine the buyer's decision to bypass after a rejection and at the seller's decision to reject an early offer. Under asymmetric information, the buyer's decision depends on her beliefs on her future demand. If $\tau$ denotes the expected value of $\theta$ conditional on the seller rejecting the offer, the buyer bypasses when

$$
\tau-k \geq \tau(1-\alpha) \Leftrightarrow \tau \geq \theta^{B} .
$$


Consider now the seller's decision to accept or reject the offer made by the buyer. This decision depends on the offer, on his information about demand, and on his beliefs about the buyer's reaction if he rejects. Indeed, after rejection, the seller obtains 0 if the buyer bypasses, whereas he obtains $\alpha \theta$ if there is no bypass and trade occurs ex post. Hence, the decision to accept or to reject a given offer $T$ depends on $\theta$ and $\tau$. This interdependency between the seller's and the buyers' actions via the belief $\tau$ may result in multiple equilibria.

For any admissible offer $T$, a seller of type $\theta$ such that $T<\theta \alpha$ rejects the early offer if he anticipates that no bypass occurs upon rejection but accepts it if he expects bypass to occur. By contrast, a seller accepts the offer in any continuation equilibrium if $\alpha \theta$ is less than $T$. Indeed, in this case, his gain under rejection can never exceed $T{ }^{6}$

The relative size of these two sets of sellers is a key determinant of the continuation equilibria following an offer $T$, which are characterized in the following lemma.

Lemma 1 Following the buyer's offer of an admissible payment $T$, the following continuation equilibria exist:

(a) Provided that the cutoff $\theta^{B}$ lies in the support of $\theta$ (i.e., $\theta^{B} \leq 1$ ), the seller accepts the offer regardless of $\theta$ and obtains a payoff of $T$. This equilibrium is supported by the buyer's out-of-equilibrium belief that the expected value of $\theta$ given rejection is not less than $\theta^{B}$, which makes bypass a credible threat.

(b) Provided that the cutoff $\theta^{B}$ is not less than $\mathbb{E}\{\theta \mid \alpha \theta>T\}$, the seller rejects the offer $T$ when it is less than $\alpha \theta$ and accepts it otherwise. The buyer believes that the expected value of $\theta$ given rejection does not exceed $\theta^{B}$ and thus does not bypass following rejection. The seller's payoff is $\max \{T, \alpha \theta\}$.

Proof. See the Appendix.

Lemma 1 highlights the difference between situations in which rejection occurs on the equilibrium path and situations in which it does not. An equilibrium in which

\footnotetext{
${ }^{6}$ Recall that with an atomless distribution over $\theta$, we do not need to specify the behavior when $\alpha \theta=T$.
} 
an early offer $T$ is accepted by all types of seller exists, provided that there are some consistent beliefs above the threshold $\theta^{B}$. Beliefs $\tau$ can then be chosen arbitrarily at any level above $\theta^{B}$; because all types of seller anticipate bypass upon rejection, accepting the offer is optimal.

In an equilibrium with no bypass, the seller rejects the offer when he knows that $\alpha \theta>T$. This requires that the buyer's updated beliefs after rejection do not induce bypass or, equivalently, that $\mathbb{E}\{\theta \mid T<\alpha \theta\} \leq \theta_{B}$.

From Lemma 1, we may distinguish three cases:

- When $\theta^{B}$ is not in the support of $\theta$ (i.e., $\theta^{B}>1$ ), bypass is never a credible threat. An early offer $T$ is therefore accepted by the seller when $T$ is greater than $\alpha \theta$ and rejected otherwise.

- When $\theta^{B}$ is smaller than $\mathbb{E}\{\theta \mid T<\alpha \theta\}$, a rejection can only induce beliefs high enough to trigger bypass. The admissible offer $T$ is therefore accepted by all types of seller in the unique continuation equilibrium.

- When $\theta^{B}$ is in the support of $\theta$ but larger than $\mathbb{E}\{\theta \mid T<\alpha \theta\}$, the two continuation equilibria of Lemma 1 exist. If the seller anticipates no bypass upon rejection, the average type of rejecting sellers is low and bypass is not profitable for the buyer. By contrast, when the seller anticipates bypass following rejection, he accepts any positive offer regardless of his type. The buyer's decision to bypass is thus consistent with out-of-equilibrium beliefs that $\theta$ is large if rejection were to occur.

\section{Equilibrium buyer's offer}

We now characterize the maximal and minimal payoffs the buyer can obtain. This will allow us to derive in a simple way the whole set of payoffs that can be sustained in equilibrium.

To begin, notice that the buyer does not make any early offer if $\theta^{B}>1$. Indeed, we have shown in this case that bypass is not a credible threat and that an early offer $T$ is accepted only if the seller obtains more than $\alpha \theta$. As a consequence, the 
expected payoff of the buyer under early contracting cannot exceed her payoff when she waits and trades ex post.

This first result illustrates that making an early offer is profitable for the buyer only when bypass is a credible threat, which requires that $\theta^{B} \leq 1$. When this is the case, there always exists an equilibrium in which the buyer captures all gains from trade.

Proposition 1 If $\theta^{B}$ is in the support of $\theta$, then there exists an equilibrium in which the buyer offers a zero price, $T=0$, and obtains all gains from trade $\mathbb{E}\{\theta\}$. In this equilibrium, all types of seller accept the offer and the buyer bypasses in the event of rejection. It is sustained by the buyer's out-of-equilibrium belief that the expected value of $\theta$ given rejection is not less than $\theta^{B}$. This is the unique equilibrium if $\theta^{B}$ is less than the mean value of $\theta$ (i.e., $\theta^{B}<\mathbb{E}\{\theta\}$ ).

Proof. See the Appendix.

Proposition 1 shows how the buyer may use the threat of bypass to shift the gains from trade to her benefit. To determine the limits of such rent shifting, we now focus on the selection rule that is the least favorable to the buyer. We also focus on the case in which $\theta^{B} \leq 1$, as otherwise the threat of bypass is never credible.

Definition 1 We define the minimal scenario as a selection rule following any admissible $T$ such that (i) if $\theta^{B}$ is greater than $\mathbb{E}\{\theta \mid T<\alpha \theta\}$, there is no bypass following rejection, and (ii) if $\theta^{B}$ is not greater than $\mathbb{E}\{\theta \mid T<\alpha \theta\}$, the offer is accepted by the seller for all $\theta$ and the buyer would bypass following rejection.

This minimal scenario is a selection rule that allows us to choose among the two possible continuation equilibria defined in Lemma 1. Under this scenario, the continuation equilibrium that prevails, on and off the equilibrium path, is the one that induces the minimal payoff to the buyer.

Under the minimal scenario, the seller does not expect bypass to occur unless $T$ is large enough. The buyer then chooses between a low $T$ rejected by some types and a high $T$ accepted by all types. We can use a logic similar to that developed for the case in which $\theta^{B}>1$ to show that the former option cannot be profitable for 
the buyer: because no bypass occurs following rejection, this would indeed require leaving to those types of seller who accept more than their gain from ex post trade (i.e., $\alpha \theta)$.

In this minimal scenario, we are then led to consider only the offers accepted by all types of seller. These offers are characterized by the condition $\mathbb{E}\{\theta \mid T<\alpha \theta\} \geq \theta^{B}$, such that the threat of bypass is credible. Among these offers, the buyer chooses the least costly offer. When $\mathbb{E}\{\theta\} \leq \theta^{B} \leq 1$, this corresponds to the price $T_{\text {min }}$ defined by

$$
\mathbb{E}\left\{\theta \mid T_{\min }<\alpha \theta\right\}=\theta^{B}
$$

Observe that the price $T_{\text {min }}$ varies with $\theta^{B}$ from 0 (when $\theta^{B}=\mathbb{E}\{\theta\}$ ) to $\alpha$ (when $\left.\theta^{B}=1\right)$. The seller therefore has two options: either she proposes an early offer $T_{\text {min }}$, or she waits and obtains the good at an expected price $\alpha \mathbb{E}\{\theta\}$. Comparing these two options leads to the next proposition.

Proposition 2 Suppose that the minimal scenario prevails and that $\theta^{B}$ lies in the support of $\theta$ but is above $\mathbb{E}\{\theta\}$. Then, the buyer makes an early offer $T_{\text {min }}$, that is accepted by the seller regardless of $\theta$, if $\theta^{B}$ is smaller than $\mathbb{E}\{\theta \mid \theta>\mathbb{E}\{\theta\}\}$. Otherwise, the buyer makes no offer.

Proof. The buyer makes an early offer $T_{\text {min }}$ if and only if it costs less than $\alpha \mathbb{E}\{\theta\}$. From equation (2), this holds when $\mathbb{E}\{\theta \mid \theta>\mathbb{E}\{\theta\}\}>\theta^{B}$.

The early offer enables the buyer to exploit information asymmetries to increase the credibility of the threat of bypass, thereby increasing her bargaining position relative to the case of symmetric information. This offer affects the sharing of the gains from trade between the seller and the buyer. Among the different types of seller, the offer creates some winners - the lower types - and some losers - the higher types. Whereas lower types accept the offer because they gain more than by trading ex post, the higher types' decision to accept depends on the anticipated consequence of their rejection.

Suppose first that the buyer proposes a low $T$. Most seller types gain more by trading ex post than by accepting this offer. Therefore, a buyer observing that her offer is rejected cannot infer much regarding the type of the seller. Suppose instead 
that the buyer proposes a high T. Most of the types gain by accepting this offer, except the higher types. A seller refusing would thus be perceived as being of a higher type and would then be bypassed by the buyer. Therefore, even if all sellers accept in fine the offer, the lower types of seller do so because it is a dominant strategy, whereas the higher types of seller accept only because refusing would trigger bypass by the buyer.

Exploiting the above logic allows the buyer to shift the division of the surplus in her favor when it is easy to make the threat of bypass credible. This is the case when $\theta^{B}$ is low, that is, when bypass is cheap or when the buyer's ex post bargaining power is weak. If instead $\theta^{B}$ is high, the threat of bypass is hardly credible and the buyer discards early contracting because it is too costly to make the threat credible.

When the buyer makes an early offer, her expected utility depends in a nonmonotonic way on the ex post bargaining power $\alpha$. Increasing the seller's bargaining power has two effects. First, the buyer's incentives to bypass increase. Second, for given incentives to bypass (thus for a fixed $\theta^{B}$ ), more types of seller reject the offer, and it therefore becomes more costly to make bypass credible. A change in the ex post bargaining power may thus benefit either the seller or the buyer.

To conclude this section, we note that Cramton and Palfrey (1995) study similar situations but from an implementation perspective. They consider a mechanismdesign problem in which agents can exercise a veto that induces a predefined default game. They define the concept of ratifiability - with two forms, weak and strong to select among multiple allocations. The equilibrium payoff of the buyer under the minimal scenario corresponds to her maximal payoff over all of the strongly ratifiable allocations. $^{7}$

\section{Equilibrium set}

We have focused thus far on two particular equilibria, one generating the maximal payoff for the buyer and another (obtained under the minimal scenario) generating

\footnotetext{
${ }^{7}$ We refer to our working paper, Jullien, Pouyet and Sand-Zantman (2013), for further discussion on this point.
} 
the minimal payoff for this buyer. Other offers can be proposed in equilibrium. Indeed, any continuation rules compatible with Lemma 1 can be considered. For instance, an offer $T=\varepsilon$, with $\varepsilon$ being positive but small, can be sustained as an equilibrium offer - accepted by all types of sellers - if (i) the buyer believes that $\theta$ is above $\theta^{B}$ when the contract $T=\varepsilon$ is rejected and (ii) the continuation equilibrium is given by the minimal scenario for any other contract offered by the buyer. Under these two conditions, the buyer cannot expect to pay less than $T_{\min }$ by deviating from the equilibrium offer and thus continues to accept offer $T=\varepsilon$. The full set of equilibrium payoffs is derived following this logic.

Proposition 3 Suppose that $\theta^{B}$ lies in the support of $\theta$. Then, the following hold:

(a) In equilibrium, the buyer obtains at least $\mathbb{E}\{\theta\}-\min \left\{\alpha \mathbb{E}\{\theta\}, T_{\text {min }}\right\}$ and at most the whole surplus $\mathbb{E}\{\theta\}$.

(b) Any admissible offer $T$ less than or equal to $\min \left\{\alpha \mathbb{E}\{\theta\}, T_{\min }\right\}$ can be sustained as an equilibrium offer accepted by all types of seller.

Proof. See the Appendix.

The above proposition shows the extent to which the buyer can gain by proposing an early contract. At worst, she can decide not to propose anything, and at best, she can reap the whole surplus. Although part (b) of the proposition highlights efficient equilibrium outcomes (that is, with no bypass), we should stress that part (a) also allows for inefficient outcomes, which exist when $T_{\min }$ is positive. ${ }^{8}$

\section{Comparison across information structures}

It is interesting to compare these equilibrium payoffs with those obtained under alternative informational structures. We consider two information structures: in the

\footnotetext{
${ }^{8}$ For instance, consider the situation in which the buyer offers $T=0$ : all types of seller accept except a small mass of types above $\theta^{B}$, and rejection triggers bypass. This offer can be supported by assuming that any other contract is followed by the minimal scenario continuation equilibrium. In the inefficient equilibria, all types of seller obtain zero surplus but the buyer does not reap the full potential gains from trade.
} 
case of incomplete and symmetric information, both the seller and the buyer know only the distribution of $\theta$. In the case of complete information, both the seller and the buyer know the value of $\theta$. In what follows, we restrict attention to the interesting case in which $\theta^{B}$ lies in the support of $\theta$, i.e., $\theta^{B} \leq 1$.

Note first that the maximal profit of the buyer under asymmetric information is the total surplus. When this arises in equilibrium, the buyer clearly benefits from asymmetric information. Henceforth, we instead focus on the minimal profit under asymmetric information and compare it with the profit obtained under the alternative information structure. This minimal profit is equal to $\mathbb{E}\{\theta\}$ if $\theta^{B} \leq \mathbb{E}\{\theta\}$ and equal to $\mathbb{E}\{\theta\}-\min \left\{\alpha \mathbb{E}\{\theta\}, T_{\text {min }}\right\}$ otherwise.

Incomplete and symmetric information. With symmetric incomplete information, the buyer makes an offer $T=0$ if $\mathbb{E}\{\theta\}>\theta^{B}$ (as shown in Section 2). The outcomes with the two information structures are thus identical, the buyer being able to reap the full surplus. If, instead, $\mathbb{E}\{\theta\}$ is less than $\theta^{B}$, there is no early offer when information is symmetric and incomplete. By contrast, under asymmetric information, the buyer offers an early contract that is to her advantage. Thus, the buyer benefits from the seller's superior information. ${ }^{9}$

Complete information. With complete information, the equilibrium outcome depends on the realized value of $\theta$. When $\theta$ is small - specifically when $\theta$ is less than $\theta^{B}$ - the buyer does not offer any early contract. By contrast, when $\theta$ is large - when $\theta$ is greater than $\theta^{B}$ - the buyer proposes $T=0$ and obtains the full surplus. ${ }^{10}$ The buyer's expected cost is therefore given by

$$
C^{C I}=\int_{0}^{\theta^{B}} \alpha \theta f(\theta) d \theta<\alpha \mathbb{E}\{\theta\},
$$

\footnotetext{
${ }^{9}$ The same conclusion holds if $\mathbb{E}\{\theta\}=\theta^{B}$. The game under incomplete and symmetric information has two equilibria ( $T=0$ and no offer), whereas $T=0$ is the unique equilibrium offer under asymmetric information.

${ }^{10}$ In the zero-probability event that $\theta=\theta^{B}$, there are two equilibria.
} 
where the inequality follows from the fact that $\theta^{B}$ is less than 1 . We know that if $\theta^{B}$ is less than $\mathbb{E}\{\theta\}$, the only equilibrium under asymmetric information is such that $T=0$. Moreover, as $T_{\min }=0$ for $\theta^{B}=\mathbb{E}\{\theta\}$, by continuity, the buyer is strictly better off under asymmetric information when $\theta^{B}$ is above but close to $\mathbb{E}\{\theta\}$. By contrast, $T_{\min }=\alpha \mathbb{E}\{\theta\}$ when $\theta^{B}$ is large, implying that the buyer is better off under symmetric information. Therefore, when $\theta^{B}$ is not too high (for instance when the cost of bypass is relatively low) but above $\mathbb{E}\{\theta\}$, the buyer prefers not to be informed if the seller is informed. ${ }^{11}$ Under complete information, the price paid by the buyer must exactly match the ex post profit for the seller with a type below $\theta^{B}$. This price is therefore high for types below but close to $\theta^{B}$. Under asymmetric information, the price paid is the same for all types of seller. Thus, asymmetric information reduces the buyer's cost for intermediate values of $\theta$ but increases that cost for small or large values. The former effect dominates when the cost of bypass is small - when $T_{\min }$ is small enough.

The main conclusion from this section is that a buyer can strictly benefit from adverse selection compared with symmetric information. This occurs when bypass is neither very credible - in which case complete information dominates for the buyer nor barely credible - in which case no information is equally good for the buyer. To illustrate this comparison across informational structures, we represent the buyer's gain as a function of $\theta^{B}$ (an increasing function of the cost $k$ ) for the case of a uniform distribution of $\theta$ over $[0,1]$.

- Insert Figure 1 approximately here -

\section{Extensions}

\section{Uncertainty regarding the seller's information}

In our model, private information on the seller's side is crucial for the buyer to benefit from early contracting. However, in some cases, the buyer may be uncertain

\footnotetext{
${ }^{11}$ In the uniform case, we can easily confirm the intuition according to which there exists a threshold $\hat{\theta}^{B}$ such that the buyer's payoff is higher under asymmetric information if and only if $\theta^{B} \leq \hat{\theta}^{B}$.
} 
of whether the seller is indeed privately informed. We examine this situation in greater detail by assuming that a positive share $\lambda$ of sellers are informed about $\theta$ whereas a share $1-\lambda$ are not; sellers in the latter group thus hold the same beliefs as does the buyer.

In the continuation equilibrium in which all types of seller accept the offer (case (a) of Lemma 1), the presence of uncertainty regarding the information set of the seller does not alter the buyer's ability to extract all of the surplus. We therefore focus on the minimal scenario, assuming again that $\mathbb{E}\{\theta\}$ is less than $\theta^{B} .{ }^{12}$

For any offer $T$, we define the set $\mathcal{R}$ of sellers that refuse this offer if they anticipate no bypass. As the buyer always offers a fixed price smaller than $\alpha \mathbb{E}\{\theta\}$, the set $\mathcal{R}$ consists of two groups of sellers: all non-informed sellers, whose ex ante beliefs of the gains from trade are given by $\mathbb{E}\{\theta\}$, and the informed sellers with types such that $\alpha \theta>T$. The average type of seller, conditional on $\theta \in \mathcal{R}$, is then given by

$$
\mathbb{E}\{\theta \mid \theta \in \mathcal{R}\}=\frac{(1-\lambda) \mathbb{E}\{\theta\}+\lambda \operatorname{Pr}(\alpha \theta>T) \mathbb{E}\{\theta \mid \alpha \theta>T\}}{1-\lambda+\lambda \operatorname{Pr}(\alpha \theta>T)}
$$

According to the same logic as above, offering an early contract is profitable only if it is accepted by all types, and this occurs whenever $\mathbb{E}\{\theta \mid \theta \in \mathcal{R}\} \geq \theta^{B}$. When $\lambda<1$, this condition no longer holds at $T=T_{\min }$. To make her threat credible again, the buyer must thus increase the price offered to the seller up to the level $T(\lambda)$ that solves the equation

$$
\frac{(1-\lambda) \mathbb{E}\{\theta\}+\lambda \operatorname{Pr}(\alpha \theta>T(\lambda)) \mathbb{E}\{\theta \mid \alpha \theta>T(\lambda)\}}{1-\lambda+\lambda \operatorname{Pr}(\alpha \theta>T(\lambda))}=\theta^{B} .
$$

Comparing $T(\lambda)$ with $\alpha \mathbb{E}\{\theta\}$, we reach the following conclusion.

Proposition 4 Suppose that the minimal scenario prevails, that $\theta^{B}$ lies in the support of $\theta$ but is above $\mathbb{E}\{\theta\}$, and that the seller is privately informed with probability $\lambda \in(0,1)$. There exists a threshold $\underline{\lambda}$ such that the following hold:

\footnotetext{
${ }^{12}$ When $\mathbb{E}\{\theta\} \geq \theta^{B}$, offering $T=0$ is the unique equilibrium for any scenario, as in the baseline model.
} 
(a) if $\lambda$ is not greater than $\underline{\lambda}$, the buyer does not offer an early contract;

(b) if $\lambda$ is greater than $\underline{\lambda}$, the buyer offers the price $T(\lambda)$ defined by (4) and the seller accepts for all $\theta ; T(\cdot)$ is decreasing with the probability that the seller is informed.

Proof. See the Appendix.

When the seller is uninformed with positive probability, a rejection does not convey as much information. The only way for the buyer to generate information that makes her threat of bypass credible is to raise the ex ante price, all the more so when the share of informed sellers is low. When $\lambda<\underline{\lambda}$, the buyer relies on ex post trade only because an early contract would require offering an excessively large payment to the seller. When the share of informed sellers is high $(\lambda \geq \underline{\lambda})$, the buyer can offer an ex ante price smaller than the expected ex post price that screens sufficiently many sellers for bypass to be credible if the offer is refused. As the likelihood that the seller is informed increases, bypass gains credibility for any given offer $T$, thereby allowing the buyer to reduce her payment to the seller.

\section{Ex ante investment}

In this section, we investigate the consequences for the seller's incentives to invest in production capacity when this investment is undertaken prior to contracting with the buyer. As emphasized by Williamson (1975), investment is likely to be suboptimal even in the absence of early contracting due to insufficient congruence between private and social incentives. One might then conjecture that early contracting exacerbates this inefficiency by increasing the buyer's ability to capture a substantive share of the value created by the investment. Focusing again on the minimal scenario and $\theta^{B} \leq 1$, we now show that this is not the case.

To this end, let us consider an initial stage during which the seller decides whether to invest. The seller's investment has a cost $\kappa<k$ and is a necessary condition for production and trade. We assume that the seller learns the value of $\theta$ before deciding whether to invest and that investment is observable. After the investment decision, the game unfolds as previously. 
As a benchmark, consider the case without early contracting. Much in the spirit of Lemma 1, we consider two situations, depending on whether there is investment on the equilibrium path. When $\theta^{B} \leq 1$, there always exists an equilibrium in which the seller does not invest. In this equilibrium, the buyer believes that $\theta$ is high if investment occurs and hence she bypasses. This out-of-equilibrium behavior deters the seller from investing in the first place.

There exists an equilibrium in which the seller invests only if $\mathbb{E}\{\theta \mid \alpha \theta>\kappa\} \leq \theta^{B}$. In this equilibrium, the seller invests whenever it is privately profitable (i.e., when $\alpha \theta \geq \kappa)$ and knowing this, the buyer decides not to bypass. ${ }^{13}$

Note that efficiency requires that the seller invests when the benefit exceeds the cost, that is, when $\theta \geq \kappa$. In the absence of early contracting, and focusing on the most efficient equilibrium, there are two reasons that the seller's investment can be suboptimal. First, when $\mathbb{E}\{\theta \mid \alpha \theta>\kappa\}>\theta^{B}$, the signal sent by the seller's investment induces the buyer to bypass, deterring any investment by the seller in the first place. Second, when $\mathbb{E}\{\theta \mid \alpha \theta>\kappa\} \leq \theta^{B}$, the seller invests but only when $\alpha \theta \geq \kappa$. As the seller does not obtain the full return on his investment $(\alpha<1)$, this investment does not occur often enough.

We now consider the effects of early contracting on the seller's incentive to invest. Assume that some types of seller invest and denote by $\hat{\theta}$ the equilibrium cut-off type such that a seller of type $\theta \geq \hat{\theta}$ invests. If $\hat{\theta}$ were large enough that $\mathbb{E}\{\theta \mid \theta>\hat{\theta}\}>\theta^{B}$, the information inferred by the buyer upon observing the seller's investment would make the threat of bypass credible. The buyer would then offer $T=0$, leaving no profit to the seller, who, anticipating this, would choose not to invest in the first place. As a consequence, investment can only occur when many types of seller invest, that is, when $\mathbb{E}\{\theta \mid \theta \geq \hat{\theta}\} \leq \theta^{B}$. In this situation, $T_{\text {min }} \geq \alpha \hat{\theta}$, where $T_{\text {min }}$ is defined by equation (2). Then, we can replicate the analysis of the main section to conclude that the buyer's optimal early contracting strategy is to offer $T_{\min }$. If $T_{\min }>\alpha \mathbb{E}\{\theta\}$, the buyer would never make such an offer, and hence, the possibility of early contracting does not change the equilibrium investment decision. If, instead,

\footnotetext{
${ }^{13}$ The buyer may or may not bypass when the seller does not invest, depending on whether it is profitable for her to do so when she knows that $\alpha \theta \leq \kappa$.
} 
$T_{\text {min }} \leq \alpha \mathbb{E}\{\theta\}$, the buyer offers an early contract that ensures a fixed revenue for the seller. As the revenue is independent of $\theta$, all types of seller invest if $T_{\min }>\kappa$ and $\mathbb{E}\{\theta\} \leq \theta^{B} .{ }^{14}$ It follows that under the minimal scenario, there is an equilibrium in which the seller invests regardless of his type, provided that $\kappa$ is small. Thus, this investment occurs more often than in the absence of early contracting.

Proposition 5 Under the minimal scenario, the investment level with early contracting is not less than the investment level without it.

Proof. If $\mathbb{E}\{\theta\}>\theta^{B}$, there is no investment with or without early contracting. If $T_{\min }>\alpha \theta$, then the possibility of early contracting has no effect. Suppose now that $\mathbb{E}\{\theta\} \leq \theta^{B}$ and that $T_{\min } \leq \alpha \theta$. If investment occurs without early contracting, we have $\mathbb{E}\{\theta \mid \alpha \theta>\kappa\} \leq \theta^{B}=\mathbb{E}\left\{\theta \mid \alpha \theta>T_{\text {min }}\right\}$. This implies that $T_{\text {min }}>\kappa$; when investment occurs for some types without early contracting, it therefore occurs for all types with early contracting.

When $\kappa$ is large, the investment decision conveys information that makes the threat of bypass credible and there is no investment by the seller, both with and without early contracting. By contrast, when $\kappa$ is small, the investment decision conveys little information and therefore leaves the buyer's bargaining position unaffected. The buyer may then offer an early contract. As already emphasized, this early contract extracts the surplus of the higher types of seller by promising larger profits to lower types of seller. As lower types are the least likely to invest, this raises the investment level.

Investment is therefore facilitated by the presence of early contracting, but this may not increase welfare. Without early contracting, there is insufficient investment, but when early contracting occurs, there is excessive investment for types $\theta$ below $\kappa$. Comparing the two situations, we find that welfare may increase or decrease depending on the values of $\kappa$ and on the ex post bargaining power $\alpha$. As an illustration, we show in the Appendix that, for small values of $\kappa$, welfare is higher under early contracting if $\alpha$ is less than $1 / 2$.

\footnotetext{
${ }^{14}$ The last inequality reflects $\mathbb{E}\{\theta \mid \theta \geq \hat{\theta}\} \leq \theta^{B}$ with $\hat{\theta}=0$.
} 


\section{General contracts}

In this section, we allow the buyer to propose more general contracts. As there are only two states of the world, any (non-stochastic) contract can be summarized without loss of generality by a payment if the buyer does not consume and another payment if she consumes. The buyer's offer can therefore be written as a menu $(T(\hat{\theta}), p(\hat{\theta}))_{\hat{\theta} \in[0,1]}$ where $T(\cdot)$ is a fixed payment and $p(\cdot)$, with $0 \leq p \leq 1$, is the price paid in the event that she buys the good.

Let $\pi(\theta)$ be the equilibrium profit a $\theta$-type seller accepting the offer:

$$
\pi(\theta)=\max _{\hat{\theta} \in[0,1]} T(\hat{\theta})+\theta p(\hat{\theta})
$$

From standard contract theory arguments (see Salanié, 1997), the buyer must propose incentive-compatible menus that are characterized by a profit $\pi(\cdot)$ with $\dot{\pi}(\theta)=$ $p(\theta)$ and $\dot{p}(\theta) \geq 0$.

A contract is admissible if it yields a non-negative profit to all types of seller (i.e., $\pi(\theta) \geq 0$ for all $\theta$ ) and if $\pi(\theta)<\alpha \theta$ for some types. ${ }^{15}$ The rejection set $\mathcal{R}$ can then be defined as follows

$$
\mathcal{R}=\{\theta \mid \pi(\theta)<\alpha \theta\}
$$

A seller of type $\theta \in \mathcal{R}$ rejects the offer if he anticipates that no bypass will occur. A seller of type $\theta \notin \mathcal{R}$ accepts the offer in any continuation equilibrium, as the profit under rejection is at most $\alpha \theta .^{16}$

Following a contract offer, a result similar to Lemma 1 can be derived: there are at most two continuation equilibria, one with bypass and one without.

The first equilibrium exists when $\theta^{B} \leq 1$ and the seller accepts the contract with probability one because rejection triggers bypass. When this continuation equilibrium prevails for all ex ante offers, the buyer can extract the full surplus $\mathbb{E}\{\theta\}$ by proposing a contract with a price equal to zero.

\footnotetext{
${ }^{15}$ The condition that $\pi(\theta)<\alpha \theta$ comes from the fact that it is never optimal for the buyer to offer a contract that yields a profit larger than the ex post profit to all types of seller.

${ }^{16}$ Notice that admissibility and convexity of $\pi(\cdot)$ imply that $\pi(\theta)=\alpha \theta$ for at most two types, and hence, indifference between accepting or not occurs with probability 0 .
} 
The second equilibrium exists when $\mathbb{E}\{\theta \mid \theta \in \mathcal{R}\} \leq \theta^{B}$ and the seller rejects the offer when $\theta \in \mathcal{R}$. In this case, rejection does not trigger bypass.

As previously, we obtain the minimal equilibrium buyer's payoff by focusing on the minimal scenario: for any contract offer $\mathcal{C}$ and rejection set $\mathcal{R}$, the selection rule is such that (i) if $\mathbb{E}\{\theta \mid \theta \in \mathcal{R}\}<\theta^{B}$, there is no bypass following rejection, and (ii) if $\mathbb{E}\{\theta \mid \theta \in \mathcal{R}\} \geq \theta^{B}$, the offer is accepted by all types of seller and the buyer bypasses following rejection.

In this minimal scenario, the buyer should never propose a contract that induces acceptance of only a (strict) subset of types because this would cost him more than trading ex post. We therefore focus on contract offers such that all types accept.

For any contract $\mathcal{C}$ and corresponding rejection set $\mathcal{R}$, the threat of bypass is credible only when $\mathbb{E}\{\theta \mid \theta \in \mathcal{R}\} \geq \theta^{B}$. To minimize her cost, the buyer offers a contract such that this constraint is binding

$$
\mathbb{E}\{\theta \mid \theta \in \mathcal{R}\}=\theta^{B}
$$

Incentive compatibility requires that the seller's gain $\pi(\cdot)$ be convex. This allows us, first, to prove that $\mathcal{R}$ is an interval $\left(\theta^{D}, 1\right]$ (see the Appendix), where $\theta^{D}$ is such that $\mathbb{E}\left\{\theta \mid \theta>\theta^{D}\right\}=\theta^{B}$. Because the profit is larger than $\alpha \theta$ for $\theta \leq \theta^{D}$ and lower for $\theta>\theta^{D}$, the slope of $\pi(\theta)$ at $\theta^{D}$ is less than $\alpha$.

This also allows us to prove that $p(\cdot)$ must be constant in equilibrium. Suppose that this is not the case and hence that the profit schedule $\hat{\pi}(\theta)$ is strictly convex. The same rejection set $\mathcal{R}$ can be generated but at a lower cost by proposing a profit function with a constant slope as illustrated in Figure 2. Indeed, the buyer can offer a linear profit with a slope equal to the slope at $\theta^{D}$ and the same value as the initial profit schedule at $\theta^{D}$. Denoting by $p^{D}$ the constant slope, this leads to a profit function $\pi(\theta)=p^{D}\left(\theta-\theta^{D}\right)+\alpha \theta^{D} \leq \hat{\pi}(\theta)$.

- Insert Figure 2 approximately here -

The buyer's best offer thus corresponds to the price $p^{D}$ that minimizes her expected cost $\mathbb{E}\{\pi(\theta)\}=p^{D}\left(\mathbb{E}\{\theta\}-\theta^{D}\right)+\alpha \theta^{D}$. 
If $\theta^{D}>\mathbb{E}\{\theta\}$, it is the highest possible price, that is $\alpha$, and hence the buyer cannot achieve more with an ex ante contract than what she achieves with no contract.

By contrast, if $\theta^{D}<\mathbb{E}\{\theta\}$, then the best price is $p^{D}=0$ along with $T=\alpha \theta^{D}=$ $T_{\min }$ and the buyer obtains more than with no contract. ${ }^{17}$ Therefore, in the minimal scenario, the simple and unique offer $T=T_{\min }$ and $p=0$ is optimal among all contracts.

To close this section, note that the maximal surplus and the minimal surplus the buyer can extract are the same as those found in the main section with an ex ante contract restricted to be a fixed price paid in advance. The set of equilibrium payoffs is therefore still characterized by Proposition 3.

\section{Concluding remarks}

This article has studied the performance of early contracting. In the absence of efficiency motives, we show that early contracting can be motivated by the desire of an uninformed party to improve its bargaining position vis-à-vis its more informed trading partner. This can be achieved with a simple contract whereby the buyer pays a fixed price at the early stage for the option to use the good later.

The context described by our model arises in situations in which a bargaining party possesses less information about her alternative options than does the other party. For instance, a firm attempting to enter a foreign market may have less information than a potential local distributor; a municipality procuring a fiber network may have less information about local demand than the telecommunications operator; and an innovator seeking commercialization may be less informed about the market potential of the product than an established producer. In each case, the uninformed party has an alternative option, self-distribution or costly in-house production, and is less informed about the value of this option than its potential trading partner.

\footnotetext{
${ }^{17}$ Allowing negative values of $p$ can decrease the cost for the buyer but requires an assumption of perfect contractibility and verifiability of the realized states of the world, here the value of the buyer's demand. We discard this possibility for the sake of realism, but this would reinforce the strength of our early contracting mechanism.
} 
The timing of bargaining affects both the opportunities and the information held by the parties. As time elapses, some options that can play a strategic role in the negotiation may disappear. In the example discussed in the introduction, having the option of providing the service internally allows the CEO to negotiate better terms with external providers. Because that option is no longer available ex post, negotiation should occur early enough that it actually improves the CEO's bargaining position. Of course, preserving some options is only useful if they are credible, which depends on the information held by the bargaining parties. Although the common wisdom is that asymmetric information harms the uninformed party, the reverse conclusion may hold in our context. If properly addressed, asymmetric information may help the uninformed party to gain credibility. As a consequence, early contracting may be more valuable under asymmetric information than under symmetric information, one reason that a newly appointed CEO may perform better than an established CEO. In the corporate literature, hiring a new CEO from outside the firm rather than an internal one is explained by managerial incentive considerations or the need for alignment between management skills and the firm's strategy (see, e.g., Casamatta and Guembel, 2010). That the newly appointed CEO arrives with poorer information concerning the firm's environment is usually perceived as a handicap (see, e.g., Reda and Wert, 2013). Our analysis suggests that this informational disadvantage may also entail a benefit, in particular when it helps to restore the firm's bargaining power vis-à-vis informed suppliers.

Our analysis also suggests that the uninformed buyer prefers to maintain relationships with previous suppliers rather than to negotiate with new and uninformed suppliers. It also sheds light on why a buyer may choose to ease information acquisition by his trading partner. ${ }^{18}$ The trading partner would, paradoxically, benefit from not receiving the information or from refusing to enter into any ex ante negotiation. When and how some agents adopt such an "empty chair" policy is another question left for future research.

\footnotetext{
${ }^{18}$ This is reminiscent of the recent literature on information design; see, for example, Taneva (2015).
} 


\section{Appendix}

Proof of Lemma 1. Consider first case (a), in which $\theta^{B} \leq 1$. There then exists some belief after rejection $\tau \in\left[\theta^{B}, 1\right]$ such that rejection triggers bypass. Moreover, in this case, if the seller refuses a contract proposed by the buyer, then he obtains zero profit. It is thus weakly optimal for the seller to accept the offer for any value of $\theta$. If all types of seller accept the offer, $\tau$ is then a consistent belief. Therefore, for $\theta^{B} \leq 1$, there is a continuation equilibrium in which the buyer bypasses after rejection.

Consider now case (b) and an equilibrium with no bypass. In such an equilibrium, all types such that $\alpha \theta>T$ optimally reject the offer whereas others accept (indifference is of measure 0). Thus $\tau=\mathbb{E}\{\theta \mid \alpha \theta>T\}$. This is an equilibrium if and only if $\tau \leq \theta^{B}$.

Proof of Proposition 1. From Lemma 1, after an offer $T=0$, there exists a continuation equilibrium such that the offer is accepted by all types of seller and the out-of-equilibrium beliefs after rejection are high enough to trigger bypass. If this continuation equilibrium prevails, then the buyer offers $T=0$ in equilibrium.

To discuss the uniqueness result, assume that $\mathbb{E}\{\theta\}>\theta^{B}$. For any admissible $\hat{T} \geq 0$, we have $\mathbb{E}\{\theta \mid \alpha \theta>\hat{T}\} \geq \mathbb{E}\{\theta\}>\theta^{B}$. From Lemma 1, the offer $\hat{T}$ is accepted with probability 1 . As a consequence, the unique equilibrium early offer is $T=0$.

Proof of Proposition 3. To verify that an offer is indeed part of an equilibrium, we have to specify a continuation equilibrium for all possible offers, which might differ on and off the equilibrium path, in a way that deters any buyer's deviation from the candidate equilibrium offer.

Notice first that any offer $T$ must be admissible, and hence positive profit for the seller, and leads to a maximal surplus of $\mathbb{E}\{\theta\}$ for the buyer.

Consider now the condition on the buyer's payoff. The necessity is immediate because the offer $T_{\min }+\varepsilon$ with $\varepsilon>0$ is accepted by all types of seller and the buyer can also make no offer. The buyer can thus ensure an expected payoff at least equal to $\mathbb{E}\{\theta\}-\min \left\{T_{\min }, \alpha \mathbb{E}\{\theta\}\right\}$. 
For sufficiency, consider the initial stage and an offer $\bar{T} \leq T_{\min }$. We construct the equilibrium by choosing the last-stage selection mapping as follows:

(i) if $T=\bar{T}$, all types of seller accept and rejection triggers bypass.

(ii) If $T \neq \bar{T}$, and $\mathbb{E}\{\theta \mid \alpha \theta>\bar{T}\} \geq \theta^{B}$, then all types of seller accept and rejection triggers bypass.

(iii) Any other offer $T$ is rejected by the seller of types $\theta>T / \alpha$, and no bypass follows a rejection.

Thus, the last-stage continuation equilibrium coincides with the minimal scenario for all offers, except for $\bar{T}$ in which case the buyer obtains all the surplus. This implies that the maximal surplus that the buyer can expect by offering $T \neq \bar{T}$ is $\mathbb{E}\{\theta\}-\min \left\{T_{\min }, \alpha \mathbb{E}\{\theta\}\right\}$. It is thus optimal to offer $\bar{T}$. Then, condition (i) ensures that the seller accepts for all $\theta$. Finally, $\theta^{B} \leq 1$ implies that bypass is credible.

Proof of Proposition 4. For any $T$ such that $\operatorname{Pr}(\alpha \theta>T) \in(0,1), \mathbb{E}\{\theta \mid \theta \in \mathcal{R}\}$ as given by (3) increases with both $T$ and $\lambda$ because $\mathbb{E}\{\theta \mid \alpha \theta>T\}>\mathbb{E}\{\theta\}$. Therefore, $T(\lambda)$ characterized by (4) is uniquely defined and decreases with $\lambda$.

As the buyer is willing to concede at most $\alpha \mathbb{E}\{\theta\}$ to the seller, let us define $\underline{\lambda}$ as follows:

$$
\frac{(1-\underline{\lambda}) \mathbb{E}\{\theta\}+\underline{\lambda} \operatorname{Pr}(\theta \geq \mathbb{E}\{\theta\}) \mathbb{E}\{\theta \mid \theta \geq \mathbb{E}\{\theta\}\}}{1-\underline{\lambda}+\underline{\lambda} \operatorname{Pr}(\theta \geq \mathbb{E}\{\theta\})}=\theta^{B} .
$$

Hence, for $\lambda<\underline{\lambda}, \mathbb{E}\{\theta \mid \theta \in \mathcal{R}\}<\theta^{B}$ for any price smaller than $\alpha \mathbb{E}\{\theta\}$ and the buyer never makes an early offer.

When $\lambda>\underline{\lambda}, T(\lambda)$ is smaller than $\alpha \mathbb{E}\{\theta\}$ and ranges from $\alpha \mathbb{E}\{\theta\}$ when $\lambda=\underline{\lambda}$ to $T_{\min }$ when $\lambda=1$.

Illustration of inefficiencies with and without early contracting. Without early contracting, the welfare loss compared with the first best (invest if and only if $\theta \geq \kappa)$ is given $A=\int_{\kappa}^{\kappa / \alpha}(\theta-\kappa) f(\theta) d \theta$. With early contracting, the loss is given by $B=\int_{0}^{\kappa}(\kappa-\theta) f(\theta) d \theta$. 
We first characterize the slope of $A$ and $B$ with respect to $\kappa$. There are given by

$$
\frac{\partial A}{\partial \kappa}=F(\kappa)+\left[\frac{1}{\alpha}\left(\frac{\kappa}{\alpha}-\kappa\right) f(\kappa / \alpha)-F(\kappa / \alpha)\right] \text { and } \frac{\partial B}{\partial \kappa}=F(\kappa)
$$

To assess the difference between $A$ and $B$, we focus on the term $D=\left[\frac{1}{\alpha}\left(\frac{\kappa}{\alpha}-\kappa\right) f(\kappa / \alpha)-\right.$ $F(\kappa / \alpha)]$. This term has the same sign as $D=\left[\frac{1}{\alpha}\left(\frac{\kappa}{\alpha}-\kappa\right) \frac{f(\kappa / \alpha)}{F(\kappa / \alpha)}-1\right]$. When $\kappa$ is close to zero,

$$
\frac{1}{\alpha}\left(\frac{\kappa}{\alpha}-\kappa\right) \frac{f(\kappa \alpha)}{F(\kappa / \alpha)}-1 \sim \frac{1}{\alpha}\left(\frac{\kappa}{\alpha}-\kappa\right) \frac{\alpha}{\kappa}-1=\frac{1}{\alpha}-2 .
$$

Therefore, for small $\kappa, D>0$ if and only if $\alpha<1 / 2$.

Rejection set in the general case. Let us show that in any equilibrium in which rejection triggers bypass, the following hold:

1. $\pi(\bar{\theta})<\alpha$;

2. $\mathcal{R}=\left(\theta^{D}, 1\right]$ with $\theta^{D} \in[0,1]$ such that $\mathbb{E}\{\theta \mid \theta \in \mathcal{R}\}=\theta^{B}$.

Recall that from the first-order incentive constraint, $\pi(\theta)-\theta \alpha$ is a convex function.

Claim 1. To show the first claim, suppose this is not the case. As $\pi(\theta)-\alpha \theta$ is convex, $\mathcal{R} \neq \varnothing$ and $\dot{\pi}(\theta)-\alpha=p(\theta)-\alpha$, it must be the case that $p(1)>\alpha$. Therefore, there exists a maximal subset of types $\theta \in\left(\theta^{\prime}, 1\right]$ such that $p(\theta)>\alpha$. Moreover, $\theta^{\prime}>0$ from the convexity of $\pi(\theta)-\alpha \theta$ and the fact that $\mathcal{R} \neq \varnothing$. At this point, it must also be the case that $\pi\left(\theta^{\prime}\right)<\alpha \theta^{\prime}$. For those types in $\left[\theta^{\prime}, 1\right]$, let us change the contract as follows: $p(\theta)=\alpha$ and $\pi(\theta)=\pi\left(\theta^{\prime}\right)+\alpha \theta-\alpha \theta^{\prime}<\alpha \theta$. This change of contract strictly benefits the buyer. Moreover, bypass in the event of rejection remains a credible threat, as $\mathbb{E}\left\{\theta \mid \theta \in \mathcal{R} \cup\left[\theta^{\prime}, 1\right]\right\}>\mathbb{E}\{\theta \mid \theta \in \mathcal{R}\} \geq \theta^{B}$. This contradicts the optimality of the contract with $\pi(\bar{\theta}) \leq \alpha$.

Claim 2. Claim 1 and the convexity of $\pi(\theta)-\alpha \theta$ imply that $\mathcal{R}$ is an interval $(\hat{\theta}, 1]$. The condition $\mathbb{E}\{\theta \mid \theta \in \mathcal{R}\} \geq \theta^{B}$ implies that $\hat{\theta} \geq \theta^{D}$ with $\mathbb{E}\left\{\theta \mid \theta>\theta^{D}\right\}=\theta^{B}$. Optimality then implies that $\hat{\theta}=\theta^{D}$. To see that, suppose instead that $\theta^{D}<\hat{\theta}$. Then, we would have $\pi\left(\theta^{D}\right)>\alpha \theta^{D}$, implying that the contract $\pi(\theta)-\varepsilon$ because $\varepsilon<\alpha \theta^{D}-\pi\left(\theta^{D}\right)$ is accepted by all types and is better for the buyer. 


\section{References}

Bebchuk, L.A. "Litigation and Settlement under Imperfect Information." RAND Journal of Economics, Vol. 15 (1984), pp. 404-415.

Casamatta, C. and Guembel, A. "Managerial Legacies, Entrenchment, and Strategic Inertia." Journal of Finance, Vol. LXV, (2010), pp. 2403-2436.

Celik, G. and Peters, M. "Equilibrium Rejection of a Mechanism." Games and Economic Behavior, Vol. 73 (2011), pp. 375-387.

Coase, R. "Durability and Monopoly." Journal of Law and Economics, Vol. 15 (1972), pp. 143-149.

Cramton, P. and Palfrey, T. "Ratifiable Mechanisms: Learning from Disagreement." Games and Economic Behavior, Vol. 10 (1995), pp. 255283.

Dana, J.D. and Spier, K. "Designing a Private Industry: Government Auctions with Endogenous Market Structure." Journal of Public Economics, Vol. 53 (1994), pp. 127-147.

Deb, R. and Said, M. "Dynamic Screening with Limited Commitment." Journal of Economic Theory, Vol. 159 (2016), pp. 891-928.

Freixas, X., Guesnerie, R., and Tirole, J. "Planning under Incomplete Information: The Ratchet Effect." Review of Economic Studies, Vol. 52 (1985), pp. 173-191.

Grossman, S. "The Informational Role of Warranties and Private Disclosure about Product Quality." The Journal of Law and Economics, Vol. 24 (1981), pp. 461-483.

Grossman, S. and Perry, M. "Perfect Sequential Equilibrium." Journal of Economic Theory, Vol. 39 (1986), pp. 97-119.

Hart, O. and Tirole, J. "Contract Renegotiation and Coasian Dynamics." Review of Economic Studies, Vol. 55 (1988), pp. 509-540. 
Jullien, B., Pouyet, J., and Sand-Zantman, W. "An Offer You Can't Refuse: Early Contracting with Endogenous Threat." TSE Working Paper, (2013) pp. 13-415.

Kessler, A.S. "The Value of Ignorance." RAND Journal of Economics, Vol. 29 (1998), pp. 339-354.

Laffont, J.J. and Martimort, D. The Theory of Incentives. Princeton, NJ: Princeton University Press, 2002.

Laffont, J.J. and Tirole, J. "The Dynamics of Incentives Contracts." Econometrica, Vol. 56 (1988), pp. 1153-1175.

Milgrom, P. "Good News and Bad News: Representation Theorems and Applications." Bell Journal of Economics, Vol. 12 (1981), pp. 380391.

Reda, J.F. and Wert, J.A. "Internal vs. External Candidates for CEO Succession." The Corporate Board. November-December (2013), pp. $1-6$.

Salanié, B. The Economics of Contracts: A Primer. Boston, MA: The MIT Press, 1997.

Skreta, V. "Optimal Auction Design under Non-Commitment." Journal of Economic Theory, Vol. 159 (2015), pp. 854-890.

Sobel, J. "Information Control in the Principal-Agent Problem." International Economic Review, Vol. 34 (1993), pp. 259-269.

Taneva, I. "Information Design," Mimeo, University of Edinburgh, 2015.

Weitzman, M. "The 'Ratchet Principle' and Performance Incentives." Bell Journal of Economics, Vol. 11 (1980), pp. 302-308.

Williamson, O. Markets and Hierarchies: Analysis and Antitrust Implications. New York, NY: Free Press, 1975.

Williamson, O. "The Economics of Governance." American Economic Review, Vol. 95 (2005), pp. 1-18. 


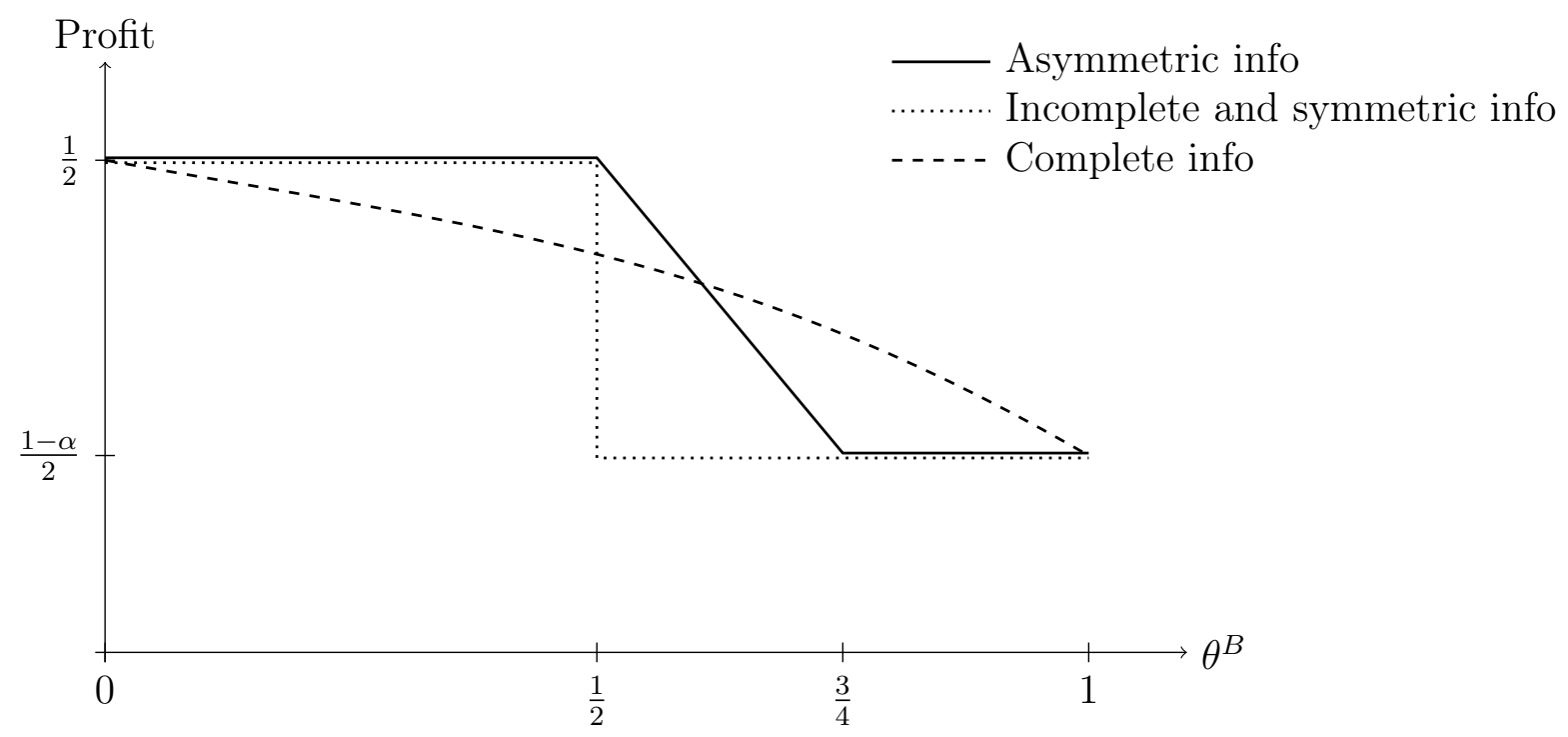

Figure 1: Buyer's expected profit as a function of $\theta^{B}$ under alternative information structures. 


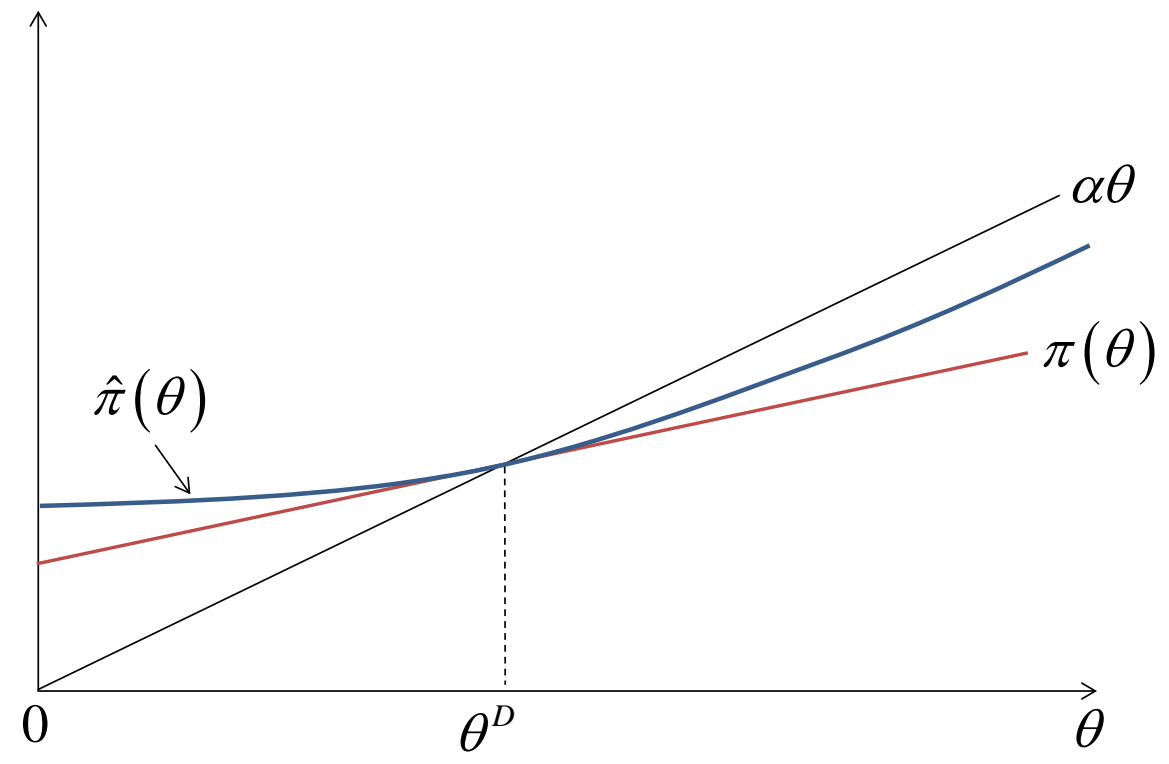

Figure 2: Optimality of linear profit functions 